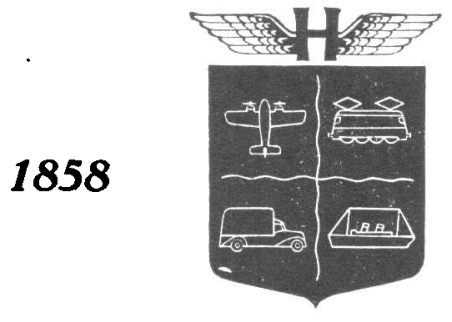

1958

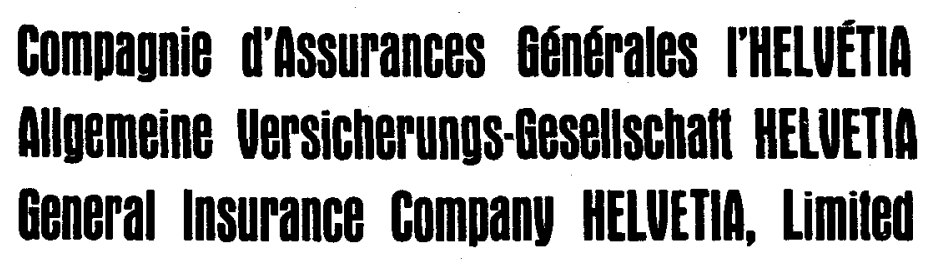

\author{
ST-GALL / ST. GALLEH \\ (SUISSE / SGHWEIL / SWITZERLANO)
}

Fondée en 1858 / Gegründet 1858 / Estd. 1858

Capital social / Aktlenkapital / Share Capital :

France sulseses

Schwolzurfranken

Swles frence

Moyens de garantie / Garantlemittel / Guarantee Funds $\quad 74.697915$

Primes brutes / Bruttoprămien / Gross Premiums 1957 : $\quad 57.675 .600$

Assurances transports et réassurances

Transportversicherungen und Ruckversicherungen

Marine Insurance and Reinsurance 


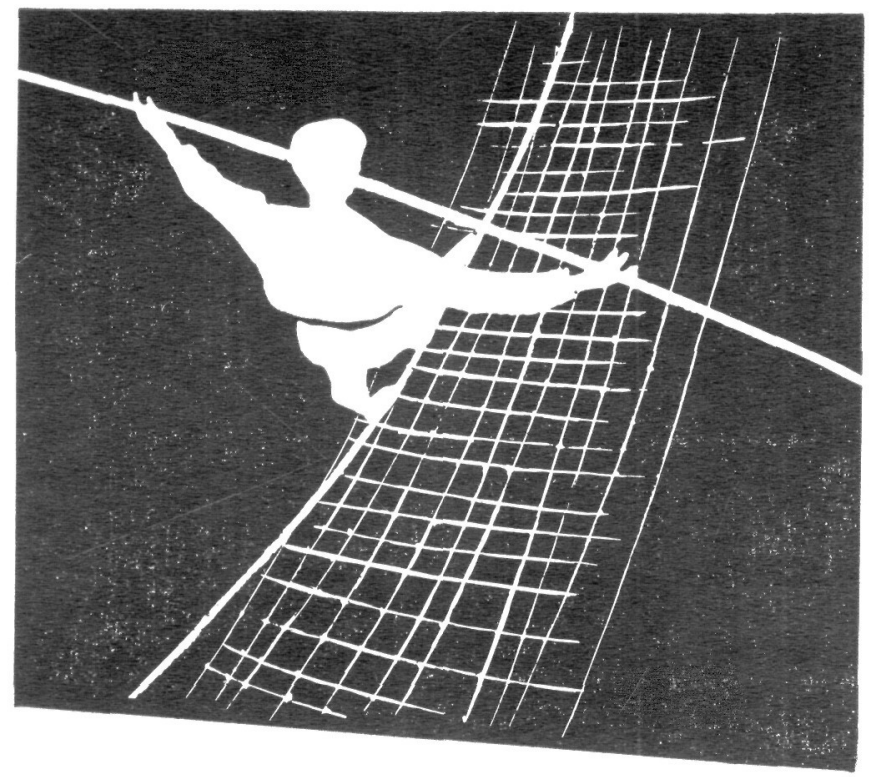

\section{La prévoyance}

\section{donne}

\section{la sécurité!}

\section{Tint puther CदEREDTIS}

- Toutes Assurances Accidents

- Responsabilité civile

- Automobiles, Casco

- Vol et Cautionnements

à des conditions avantageuses 

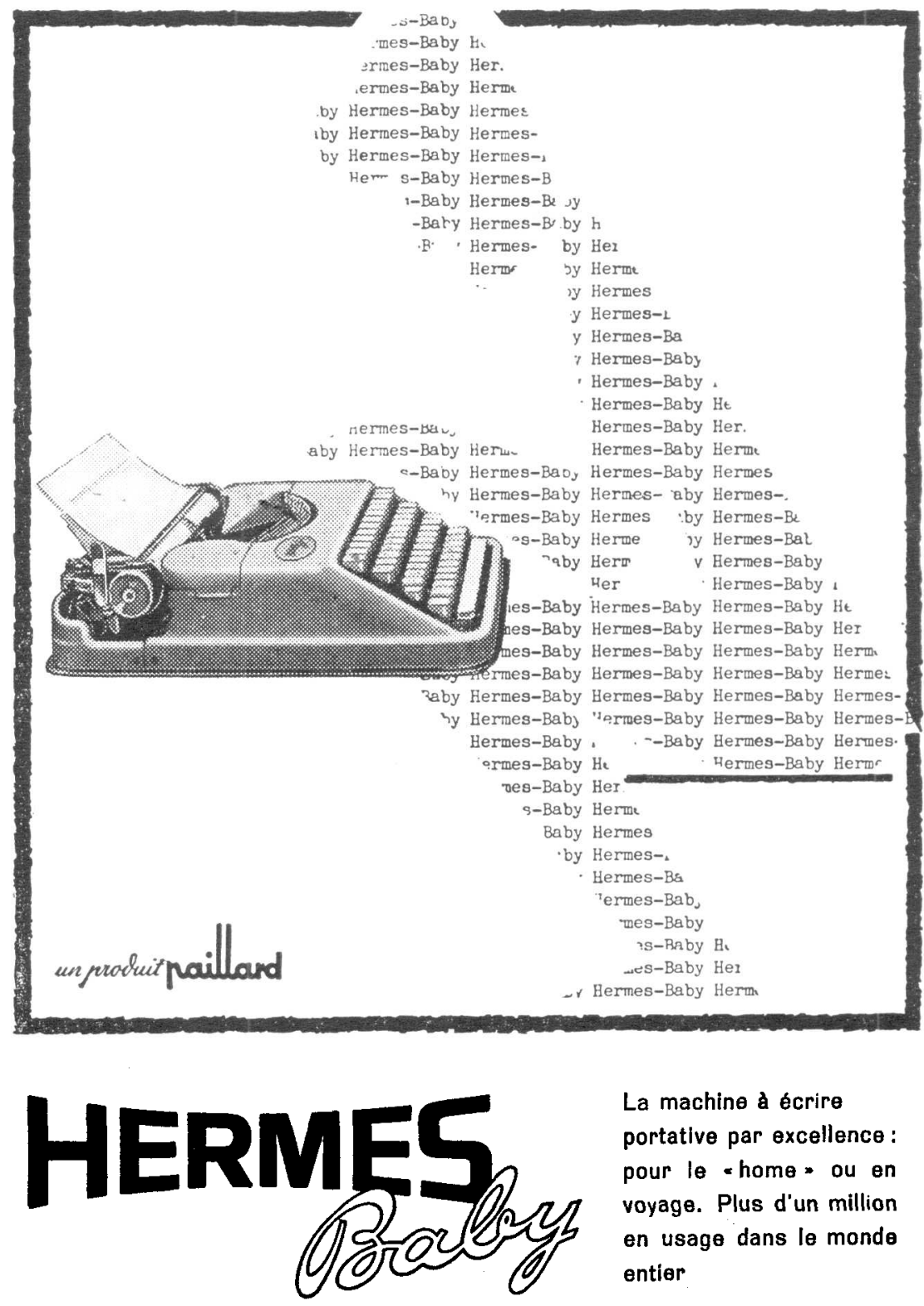

La machine à écrire portative par excellence : pour le chome* ou en voyage. Plus d'un million en usage dans le monde entior

\section{Fabricants PAILLARD S.A., Yverdon/Suisse}




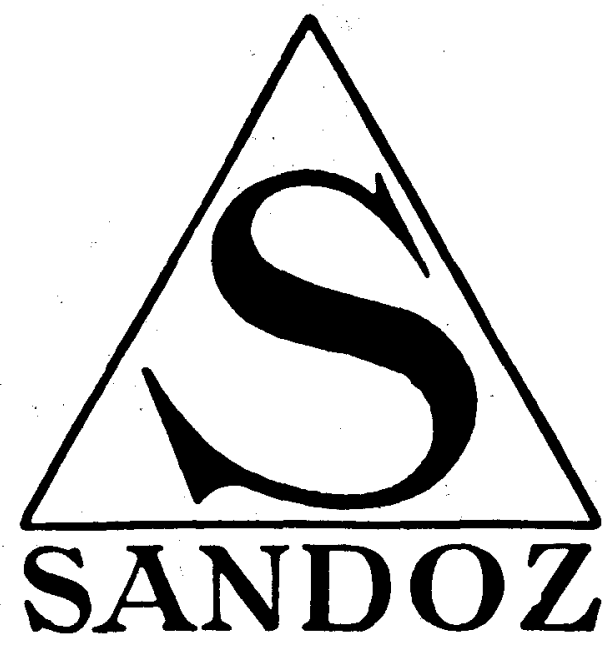

\section{PRODUITS}

\section{PHARMACEUTIQUES}

\section{BALE (Suisse)}




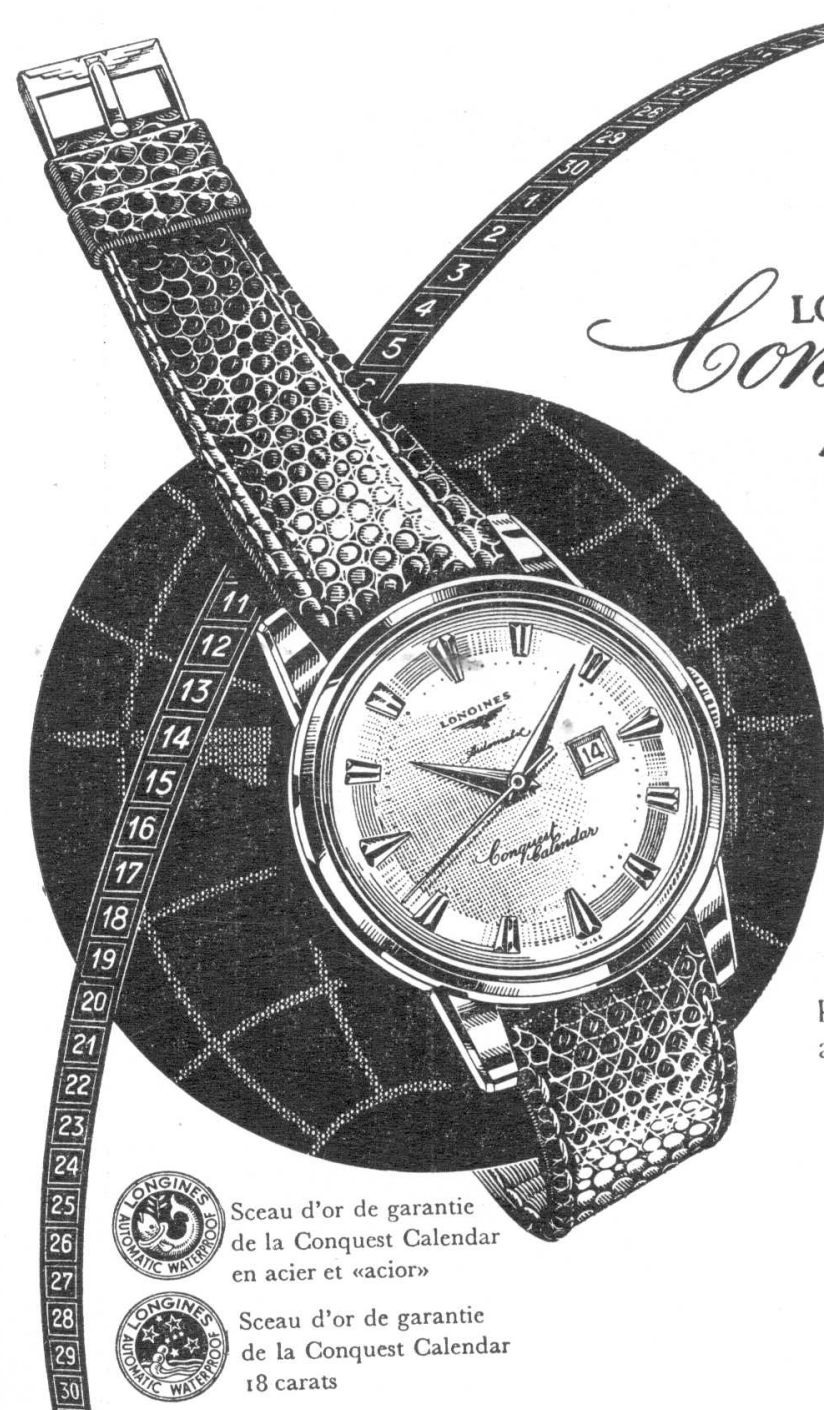




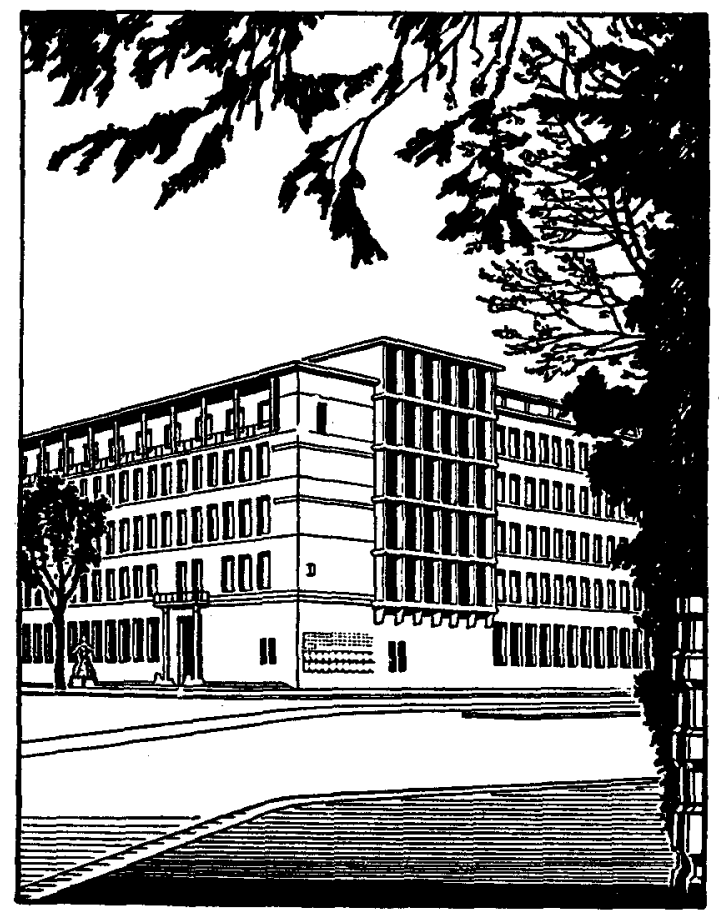

Siège à Zurich, Alpenquai 40

\section{Sociêté suisse \\ d’Assurances génépales sur la vie humaine RENTENANSTALT}

Entreprise mutuelle

Fondée en 1857

Agences générales à :

Aarau, Bale, Berne, Bienne, Coire, Fribourg. Genève, Glaris, Lausanne, Lugano, Lucerne, Neuchatel, Romanshorn, St-Gall, Sion, Soleure, Zurich, Sièges spéciaux à :

Munich - Paris - Amsterdam - Bruxelles

La plus ancienne et la plus grande des sociétés suisses d'assurances sur la vie 


\section{Canadiennes et Vêtements de travail}

confection très soignée, grand stock disponible, exécution rapide de toutes commandes, exportation pour tous pays. Veuillez demander les offres à notre bureau de vente

MONTEX S.A., 4, rue du Mont-Blanc, GENEVE, Suisse.

Câbles: Efimexport, Genève Téléphone: (022) 326612

\section{SOCIÉTÉ FIDUCIAIRE ROMANDE OFOR S.A.}

- Revisions et organisations comptables

- Administration et liquidation de sociétés

- IMPOTS: Conseils par spécialiste

9, RUE D'ITALIE - GENEVE TÉLÉPHONE 24 32 91/3

\section{RECOSEN \\ ROBAPHARM \\ BALE}

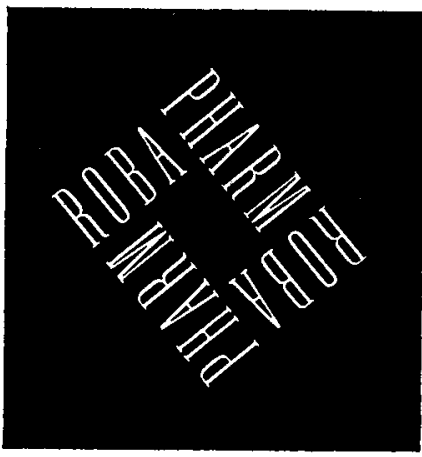



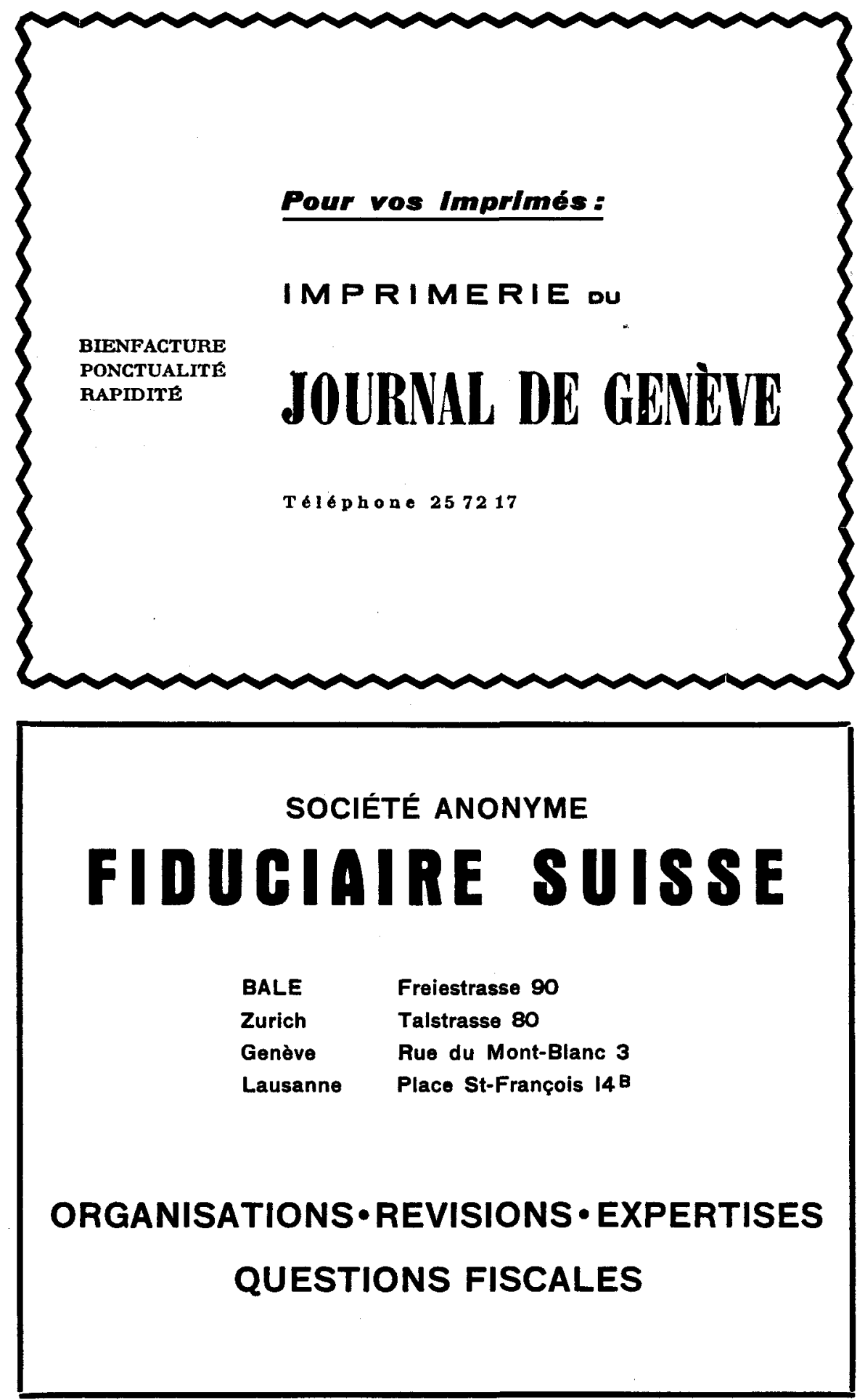


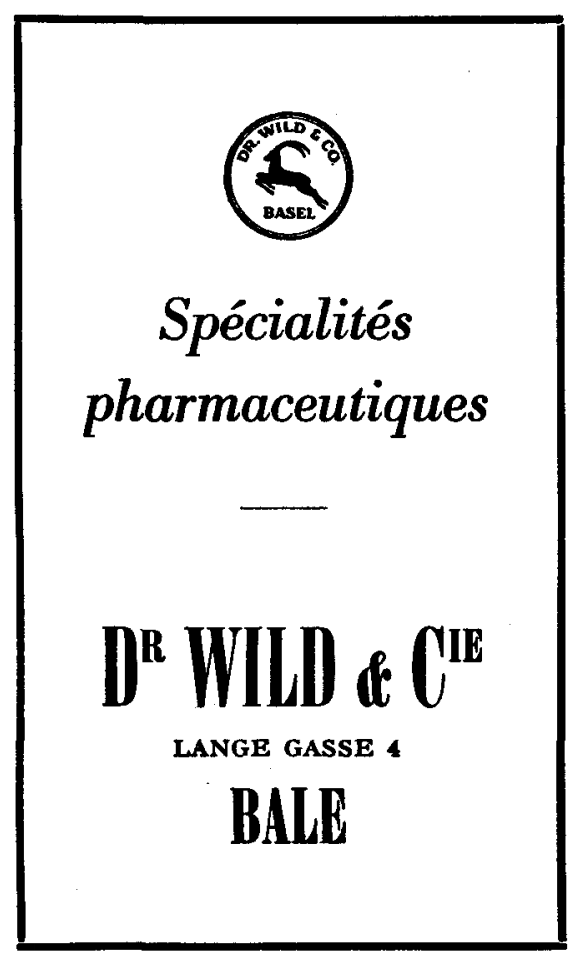

\section{LABDRATORESS VIFOR}

S.A.

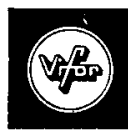

PRODUITS PHARMACEUTIQUES

GENĖVE (SUISSE)

Laboratoires

\section{SAPOS S.A.}

Spécialités pharmaceutiques

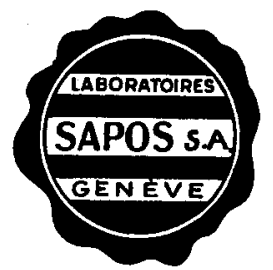

RUE GUSTAVE-MOYNIER 5

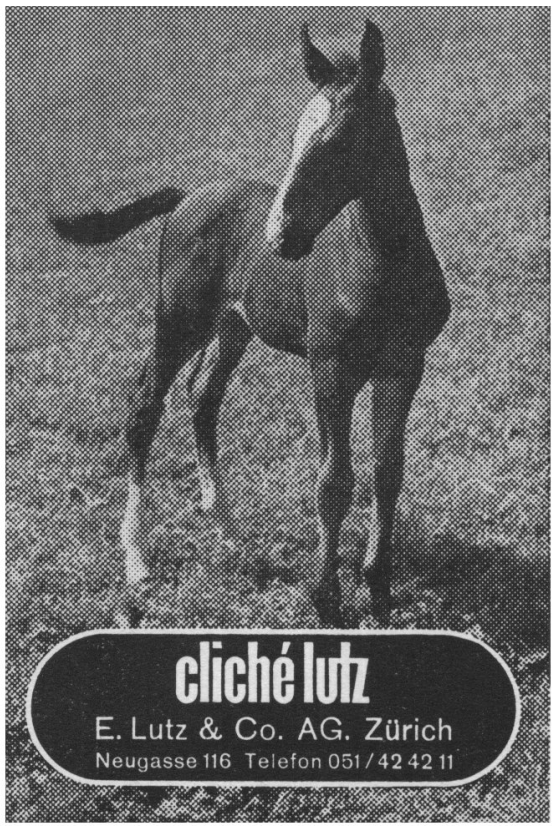




\section{BONNES ADRESSES EN SUISSE}

\section{LA RÉSIDENCE}

Florissant 11

GENEVE

Hôtel - Restaurant - Bar

Grands et petits salons pour réceptions 200 lits

60 salles de bains

Téléphone dans toutes les chambres

Deux tennis - Parc pour autos

Arrangements pour familles

Tél. 241380 (8 lignes)

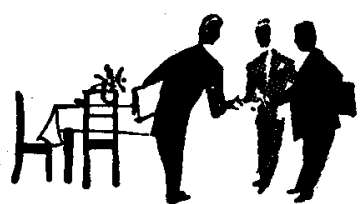

Pour leurs conférences, ils se réunissent toujours au

\section{Buffer de la Gare à berne}

\section{F. E. KRÄHENBƯHL} Téléphone 23421

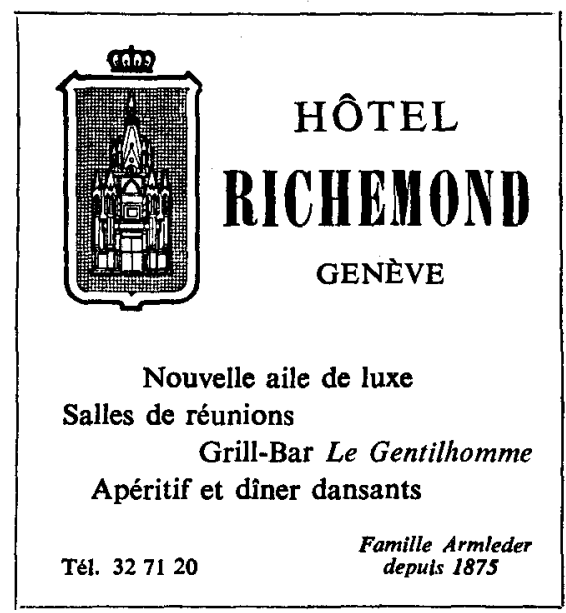

\section{Hôtel du Phône GEN E VE}

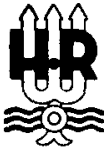

Le plus récent, construit en 1950, en plein centre. Situation tranquille au bord du Rhône, quai Turrettini. 200 chambres, toutes avec salle de bain, douches ou cabinet de toilette et radio.

Garage dans la maison Agence de voyages.

Prix à partir de fr. 16,-, chambre, petit-déjeuner et service compris.

T61. 327040 Tólégr.: RHOHOTEL 


\section{EXTRAIT DES STATUTS \\ DU COMITÉ INTERNATIONAL DE LA CROIX-ROUGE (adoptés le 25 septembre I952)}

Article premier. - Le Comité international de la Croix-Rouge (CICR), fondé à Genève en 1863 , consacré par les Conventions de Genève et par les Conférences internationales de la Croix-Rouge, est une institution indépendante ayant son statut propre.

Il est partie constitutive de la Croix-Rouge internationale ${ }^{1}$.

ART. 2. - En tant qu'association régie par les articles 60 et suivants du Code civil suisse, le CICR possède la personnalité civile.

ART. 3. - Le CICR a son siège à Genève.

Il a pour emblème la croix rouge sur fond blanc. Sa devise est "Inter arma caritas $n$.

ART. 4. - Le CICR a, notamment, pour rôle :

a) de maintenir les principes fondamentaux et permanents de la CroixRouge, à savoir : l'impartialité, une action indépendante de toute considération raciale, politique, confessionnelle ou économique, l'universalité de la Croix-Rouge et l'égalité des Sociétés nationales de la Croix-Rouge;

b) de reconnaître toute Société nationale de la Croix-Rouge nouvellement créé ou reconstituée et répondant aux conditions de reconnaissance en vigueur, et de notifier cette reconnaissance aux autres Sociétés nationales;

c) d'assumer les tâches qui lui sont reconnues par les Conventions de Geneve, de travailler à l'application fidele de ces Conventions et de recevoir toute plainte au sujet de violations alléguees des Conventions humanitaires;

d) d'agir, en sa qualité d'institution neutre, spécialement en cas de guerre, de guerre civile ou de troubles intérieurs; de s'employer en tout temps à ce que les victimes militaires et civiles desdits conflits et de leurs suites directes reçoivent protection et assistance, et de servir, sur le plan humanitaire, d'intermédiaire entre les parties;

e) de contribuer, en vue desdits conflits, à la préparation et au développement $\mathrm{du}$ personnel et du matériel sanitaires, en collaboration avec les organisations de la Croix-Rouge et les Services de santé militaires et autres autorités compétentes;

f) de travailler au perfectionnement du droit international humanitaire, à la compréhension et la diffusion des Conventions de Genève et d'en préparer les développements éventuels;

g) d'assumer les mandats qui lui sont confiés par les Conférences internationales de la Croix-Rouge.

Le CICR peut en outre prendre toute initiative humanitaire qui entre dans son rôle d'institution spécifiquement neutre et indépendante et étudier toute question dont l'examen par une telle institution s'impose.

ART. 6 (alinéa premier). - Le CICR se recrute par cooptation parmi les citoyens suisses. Le nombre de ses membres ne peut dépasser vingtcinq.

1 La Croix-Rouge internationale comprend les Sociétés nationales de la Croix-Rouge, le Comité international de la Croix-Rouge et la Ligue des Sociétés de la Croix-Rouge. L'expression - Sociétés nationales de la Croix-Rouge " couvre également les Sociétés du Croissant-Rouge et la Société du Lion et Soleil Rouges. 


\section{ADRESSES DES COMITÉS CENTRAUX}

AFGHANISTAN - Croissant-Rouge afghan, Kaboul.

ALBANIE - Croix-Rouge albanaise, 35, Rruga e Barrikadavet, Tirana.

ALLEMAGNE (République fédérale) - Croix-Rouge allemande dans la République fédérale, 71, Friedrich-EbertAllee, Bonn.

ALLEMAGNE (République démocratique) - Croix-Rouge allemande dans la République démocratique, 11, Kaitzerstrasse, Dresde.

ARGENTINE - Croix-Rouge argentine, Yrigoyen 2068 (R.72), Buenos Aires.

AUSTRALIE - Croix-Rouge australienne, 122-128, Flinders Street, Melbourne.

AUTRICHE - Croix-Rouge autrichienne, 3, Gusshausstrasse, Vienne IV.

BELGIQUE - Croix-Rouge de Belgique, 98, Chaussée de Vleurgat, Bruxelles.

BIRMANIE - Croix-Rouge de Birmanie, Strand Road Rangoon.

BOLIVIE - Croix-Rouge bolivienne, 185, Avenue Simon-Bolivar 136, La Paz.

BRÉSIL - Croix-Rouge brésilienne, Praça da Cruz Vermelha, 10-12, Rio de Janeiro.

BULGARIE - Conseil central de la Croix-Rouge bulgare, 1, boul. S.S. Biruzov, Sofia.

CANADA - Croix-Rouge canadienne, 95, Wellesley Street East, Toronto, 5.

CEYLAN - Croix-Rouge de Ceylan, 106, Turret Road, Colombo VII.

CHILI - Croix-Rouge chilienne, Avenida Santa Maria, 0150 Correo, 15, Casilla 246. V., Santiago de Chile.

CHINE - Croix-Rouge chinoise, 22 Kammein Hutung, Pékin.

COLOMBIE - Croix-Rouge colombienne, Carreraa, 7 No. 34 65, Apartado nacional 1110, Bogota.

CORÉE (République de) - Croix-Rouge de la République de Corée, 32-3 Ka Nam San Dong, Séoul.

COREE (République démocratique de) - Croix-Rouge de la République démocratique populaire de Corće, Pyongyang,

COSTA-RICA - Croix-Rouge costaricienne, Calle 5a Sur Apartado 1025, San José.

CUBA - Croix-Rouge cubaine, Ignacio Agramonte, 461, La Havane.

DANEMARK - Croix-Rouge danoise, Platanvej 22, Copenhague $V$.

RÉPUBLIQUE DOMINICAINE - Croix-Rouge dominicaine, Calle Galvan, 24, A partado 1288, Ciudad Trujillo.

EGYPTE - Société nationale du Croissant-Rouge égyptien, rue Ramsis, 34, Le Caire.

EQUATEUR - Croix-Rouge équatorienne, Avenida Colombia y Elizalde, 118, Quito.

ESPAGNE - Croix-Rouge espagnole, Eduardo Dato, 16, Madrid.

ÉTATS-UNIS - Croix-Rouge américaine. National Headquarters, 17th and D. Streets. N.W., Washington 13, D.C.

ÉTHIOPIE - Croix-Rouge éthiopienne, P.O. Box 195, Addis- $A$ béba.

FINLANDE - Croix-Rouge de Finlande, Tehtaankatu I A, Helsinki.

FRANCE - Croix-Rouge française, 17, rue Quentin-Bauchart, Paris (8me).

GHANA - Croix-Rouge du Ghana, P.O. Box 835, Accra.

GRANDE-BRETAGNE - Croix-Rouge britannique, 14, Grosvenor Crescent, Londres S.W.1.

GRECE - Croix-Rouge hellénique, rue Mackenzie-King, 1 et rue Solonos, 39 , Athenes.

GUATÉMALA - Croix-Rouge du Guatémala, 4a. C11-42, Zona No. 1, Guatémala.

HAITTI - Croix-Rouge haïtienne, rue Férou, Port-au-Prince.

HONDURAS - Croix-Rouge de Honduras, Calle Henry+ Dunant, Tegucigalpa.

HONGRIE - Croix-Rouge hongroise, Arany Janos utca, 31, Budapest $V$.

INDE - Croix-Rouge de l'Inde, Red Cross Road, New Delhi, 2.

INDONESIE - Croix-Rouge indonésienne, Tanah Abang Barat, 66, Djakarta.
IRAK - Croissant-Rouge de l'Irak, Bagdad.

IRAN - Société du Lion et Soleil Rouges de l'Iran, Avenue Ark, Téhéran.

IRLANDE - Croix-Rouge irlandaise, 25 Westland Row, Dublin.

ISLANDE - Croix-Rouge irlandaise. Thorvaldsens-straeti, 6, Reykjavik.

ITALIE - Croix-Rouge italierne, 12, Via Toscana, Rome.

JAPON - Croix-Rouge du Japon, 5, Shiba Park, Minato-Ku. Tokio.

JORDANIE - Croissant-Rouge jordanien, Amman.

LAOS - Croix-Rouge lao, Vientiane

LIBAN - Croix-Rouge libanaise, Beyrouth.

LIBERIA - Croix-Rouge du Liberia, Ducor Hall, Iog Front Street, Monrovia.

LIBYE - Croissant-Rouge libyen, Gasser Hamed Str. 14 P.O.B 239. Benghasi.

LIECHTENSTEIN - Croix-Rouge du Liechtenstein, Vadus.

LUXEMBOURG - Croix-Rouge luxembourgeoise, Parc de la Ville, Luxembourg.

MAROC - Croissant rouge marocain, 84, Bd Joffre Casablanca

MEXIQUE - Croix-Rouge mexicaine, Sinaloa, No $20,4^{\circ}$ piso Mexico DF.

MONACO - Croix-Rouge monégasque, Quai des Etats-Unis Monaco.

NICARAGUA - Croix-Rouge du Nicaragua, 12, Avenido Nordeste Managua D.N.C.A.

NORVEGE - Croix-Rouge de Norvège Parkveien, 33b, Oslo.

NOUVELLE-ZELANDE - Croix-Rouge néo-zélandaise, 61, Dixon Street, Wellington C.I.

PAKISTAN - Croix-Rouge du Pakistan, Frere Street, Karachi 4.

PANAMA - Croix-Rouge de Panama, Panama.

PARAGUAY - Croix-Rouge paraguayenne, Calle André Barbero y Artigas, Asuncion.

PAYS-BAS - Croix-Rouge néerlandaise, 27, Prinsessegracht, La Haye.

PEROU - Croix-Rouge péruvienne, Tarapaca 881, Lima.

PHILIPPINES - Croix-Rouge philippine, 600 Isaac Peral Street, P.O. Box 280, Marille.

POLOGNE - - Croix-Rouge polonaise, Mokotowska, 14. Varsovie.

PORTUGAL - Croix-Rouge portugaise, Secrétariat général. Jardim 9 de Abril, 1, Lisbonne.

ROUMANIE - Croix-Rouge de la République populaire roumaine, Strada Biserica Amzei, 29, Bucarest.

SAINT-MARIN - Croix-Rouge de Saint-Marin, Saint-Marin.

SALVADOR - Croix-Rouge du Salvador, 3a, Avenida Norte y 3a, Calle Poniente, San Salvador.

SOUDAN - Croissant-Rouge soudanais, P.O. Box 235, Khartoum.

SUEDE - Croix-Rouge suédoise, Artillerigatan, 6, Stockholm 14.

SUISSE - Croix-Rouge suisse, Taubenstrasse, 8 , Berne.

SYRIE - Croissant-Rouge syrien, Damas.

TCHÉCOSLOVAQUIE - Croix-Rouge tchécoslovaque, Thunovska, 18, Prague III.

THAiLANDE - Croix-Rouge thailandaise, King Chulalong, korn Memorial Hospital, Bangkok.

TUNISIE - Croissant-Rouge tunisien, 1, avenue de Carthage, Tunis.

TURQUIE - Société du Croissant-Rouge turc, Yenisehir Ankara.

UNION SUD-AFRICAINE - Croix-Rouge sud-africaine, Barclay's Bank Building, 14 Hollard Street, Johannesburg.

U.R.S.S. - Alliance des Sociétés de la Croix-Rouge et du Croissant-Rouge de l'U.R.S.S. Kouznetsky Most, 18/7, Moscou.

URUGUAY - Croix-Rouge uruguayenne, Juan D. Jackson, 1301, Montevideo.

VENEZUELA - Croix-Rouge vénézuélienne, Avenida de Andrés Bello, No. 4, Caracas.

VIETNAM (République du) - Croix-Rouge de la République du Vietnam, rue Hong Thap Tu, No 201, Saigon.

VIETNAM (République démocratique du) - Croix-Rouge de la République démocratique du Vietnam, 68 , rue BaTriez, Hanoi.

YOUGOSLAVIE - Croix-Rouge yougoslave, Simina ulica broj, 19, Belgrade. 